\title{
Multistability of Phase-Locking and Topological Winding Numbers in Locally Coupled Kuramoto Models on Single-Loop Networks
}

\author{
Robin Delabays, Tommaso Coletta, and Philippe Jacquod \\ School of Engineering, University of Applied Sciences of Western Switzerland, CH-1950 Sion, \\ Switzerland
}

(Dated: 14 December 2015, revised: 15 February 2016, published: 9 March 2016)

\begin{abstract}
Determining the number of stable phase-locked solutions for locally coupled Kuramoto models is a long-standing mathematical problem with important implications in biology, condensed matter physics and electrical engineering among others. We investigate Kuramoto models on networks with various topologies and show that different phase-locked solutions are related to one another by loop currents. The latter take only discrete values, as they are characterized by topological winding numbers. This result is generically valid for any network, and also applies beyond the Kuramoto model, as long as the coupling between oscillators is antisymmetric in the oscillators' coordinates. Motivated by these results we further investigate loop currents in Kuramoto-like models. We consider loop currents in nonoriented n-node cycle networks with nearest-neighbor coupling. Amplifying on earlier works, we give an algebraic upper bound $\mathcal{N} \leq 2 \operatorname{Int}[n / 4]+1$ for the number $\mathcal{N}$ of different, linearly stable phase-locked solutions. We show that the number of different stable solutions monotonically decreases as the coupling strength is decreased. Furthermore stable solutions with a single angle difference exceeding $\pi / 2$ emerge as the coupling constant $K$ is reduced, as smooth continuations of solutions with all angle differences smaller than $\pi / 2$ at higher $K$. In a cycle network with nearest-neighbor coupling we further show that phase-locked solutions with two or more angle differences larger than $\pi / 2$ are all linearly unstable. We point out similarities between loop currents and vortices in superfluids and superconductors as well as persistent currents in superconducting rings and two-dimensional Josephson junction arrays.
\end{abstract}

PACS numbers: 05,45.-a, 05.45.Xt, 84.70.+p

Keywords: Kuramoto model, multistability, winding numbers, loop flows

\section{INTRODUCTION}

From large colonies of fireflies flashing in unison to single-frequency electric power grids covering areas as large as entire continents, from human brain waves to arrays of submicronic Josephson junctions, there are many, disparate systems that exhibit collective synchrony ${ }^{1}$. Following early works, most notably by Winfree ${ }^{2}$, a window towards a quantitative, mathematical understanding of collective synchrony was opened by Kuramoto ${ }^{3}$ who proposed a model of coupled oscillators defined by the following set of nonlinear differential equations

$$
\dot{\theta}_{i}=P_{i}-\sum_{j=1}^{n} K_{i j} \sin \left(\theta_{i}-\theta_{j}\right), \quad i=1, \ldots n
$$

The model describes the dynamics of a set of $n$ one-dimensional oscillators with angular coordinates $\theta_{i}$ and natural frequencies $P_{i}$ under the influence of a coupling that is periodic in their angle differences. 
The Kuramoto model has become a standard model for investigating the transition to synchrony in coupled dynamical systems $\underline{4} \underline{-6}$.

The beauty of the Kuramoto model is that it is sufficiently simple to allow for analytical treatments of the emergence of synchrony in coupled oscillators systems, while retaining most of the essence of this complex problem. As a matter of fact, Kuramoto observed early on that for constant all-to-all coupling, $K_{i j} \equiv K / n$, an analytically solvable mean-field solution becomes exact in the large $n$ limit. A coherent, synchronous state $\left\{\theta_{i}^{(0)}\right\}$ emerges for $K>K_{c}$ in the form of a solution to Eq. (1.1) with $\dot{\theta}_{i}^{(0)}-\dot{\theta}_{j}^{(0)}=0$, for at least a finite fraction of pairs of oscillators $(i, j)$. The critical coupling strength $K_{c}$ depends on the distribution $g(P)$ of natural frequencies $P_{i}$, and phase-locking with $\dot{\theta}_{i}^{(0)}-\dot{\theta}_{j}^{(0)}=0$, for all $i, j$, can be achieved if $g(P)$ has compact support $\underline{7.8}$.

Most physical systems exhibiting synchrony consist however in collections of subsystems with shortrange coupling. The problem becomes much more complicated for Kuramoto models defined on such complex networks with reduced node degree because the mean-field approach no longer applies. Phaselocked solutions $\left\{\theta_{i}^{(0)}\right\}$ to Eq. (1.1) are determined by

$$
P_{i}=\sum_{j=1}^{n} K_{i j} \sin \left(\theta_{i}^{(0)}-\theta_{j}^{(0)}\right),
$$

i.e. by a set of $n$ nonlinear algebraic equations which, in principle, accept more than one solution. For instance new solutions to Eq. (1.2) can be obtained from known solutions by substituting $\theta_{i}^{(0)}-\theta_{j}^{(0)} \rightarrow$ $\pi-\left(\theta_{i}^{(0)}-\theta_{j}^{(0)}\right)$ for some or all $(i, j)$. This can lead, in principle, to an exponential number $\propto 2^{n}$ of solutions, however, many of them are not dynamically stable in the sense given by Eq. (1.1). It has in particular been shown that there is a single stable solution above the transition to synchrony for all-to-all couplings 9,10 and for identical oscillators $\left(P_{i}=\bar{P}\right.$, for all $\left.i\right)$ on networks with sufficiently large node degree 11 .

To be physically relevant, a solution of Eq. (1.2) needs to be robust against any small perturbation. Thus, the truly important question is "how many linearly stable phase-locked solutions to the Kuramoto problem are there ?" This question dates back at least to the work of Korsak ${ }^{12}$ in the context of the power flow problem (dealing in particular with conditions for operational synchrony in the electric power grid) 13 , which is closely related to the Kuramoto model. As a matter of fact, it turns out (see below in Section $\amalg \mathrm{A}$ ) that for high voltages, a first approximation is to neglect ohmic losses, in which case AC electric power transport between the nodes of a power grid is governed by Eq. (1.2), with $P_{i}$ being the power injected $\left(P_{i}>0\right)$ or extracted $\left(P_{i}<0\right)$ at node $i$. Korsak provided a simple example of a network where different, linearly stable solutions exist that differ by some circulating loop current. Similar works have dealt with that problem since then, motivated by issues of voltage and phase stability that are central to the stable, synchronous operation of electric power grids. Most, if not all of these investigations are however restricted to numerical investigations on small networks. The literature on the subject is rather large and we refer the interested reader to Refs. 14 and 15 for more information. Bounds for the number of different stable solutions were first constructed in the spirit of the argument given after Eq. (1.2). In this way, Refs. 16 and 17 gave an exponential upper bound for the number of power flow solutions, as did Ref. 18 for the phase-locked solutions of the Kuramoto model. Ref. 18 observed numerically, however, that the number of stable solutions is much smaller than $2^{n}$. Below we show that a much better, algebraic upper bound is obtained when considering that quantized loop currents differentiate between different stable solutions.

To the best of our knowledge, the characterization of loop flows with topological winding numbers has been first made by Janssens and Kamagate ${ }^{19}$, though Lüders (in a referee discussion at the end of Ref. 12) and Ermentrout $\underline{\underline{7}}$ already point to it. Topological winding numbers emerge from the 
consistency requirement that summing angle differences along any cycle in a network must give an integer multiple of $2 \pi$. Below we illustrate how this leads to loop currents that can take only discrete values. Similar considerations in a different physical context lead to the quantization of circulation around vortices in a superfluid 20,21 or a type-II superconductor ${ }^{22}$ and to the quantization of persistent currents in superconducting rings ${ }^{23,24}$ and rings of Josephson junctions $\frac{25}{2}$. That such a similarity exists is not a surprise, given that each term on the right-hand-side of Eq. (1.2) gives the Josephson current between two superconductors with order parameter $\psi_{i, j}=n_{s}^{1 / 2} \exp i \theta_{i, j}^{(0)}$ coupled by a tunnel junction of transparency $T_{i j}=\hbar K_{i j} / 8 e n_{s}$. More surprising, however, is that investigations on small cycle networks with injections and consumptions show that loop currents persist even in networks with ohmic dissipation 12,26 .

Using winding numbers, Rogge and Aeyels 27 obtained an algebraic upper bound $\mathcal{N} \leq 2 \operatorname{Int}[n / 4]+1$ for the number of stable solutions with any angle difference in a Kuramoto model on a $n$-node ring with unidirectional nearest-neighbor couplings. The same upper bound has been calculated by Ochab and Góra ${ }^{28}$ in a nonoriented $n$-node Kuramoto ring with nearest-neighbor coupling, under the condition that all angle differences are smaller than $\pi / 2$. This upper bound is reached when the coupling strength goes to infinity, equivalently corresponding to $P_{i}=0$, for all $i$ in Eqs. (1.1) and (1.2), i.e. to identical oscillators. This alternatively gives the number of stable states for Josephson junction rings in the classical regime, neglecting Coulomb interaction effects ${ }^{24}, 25$. Different solutions for the Kuramoto model where investigated semi-analytically in Ref. 29 and classified according to two integers, $q$ (the winding number mentioned above) and $l$ (the number of angle differences larger than $\pi / 2$ ) in Ref. 30 . In a somewhat different but related direction of investigation, Wiley et al ${ }^{31}$ investigated the size of the basin of attraction for synchronous solutions with different $q$ in a cycle network of identical oscillators and found that it gets smaller at higher $q$.

Below we show that two different solutions to Eq. (1.2) on any network differ only by loop currents. This provides additional motivation for investigating loop currents as it rigorously connects them to multiple stable solutions to Eq. (1.2). We thus investigate single-loop networks and show that the algebraic upper bound of Rogge and Aeyels $\frac{27}{}$ and Ochab and Góra 28 is generically valid for the Kuramoto model on a nonoriented cycle with nearest-neighbor interactions. We furthermore demonstrate that, for such networks, at most one angle difference can exceed $\pi / 2$. Stable solutions are in particular restricted to only $l=0$ or $l=1$ in the classification scheme of Ref. 30 . We show that the number of stable solutions decreases monotonically as the coupling strength is reduced, and that solutions with $l=1$ emerge continuously at lower coupling from solutions with $l=0$.

The manuscript is organized as follows: Section 1 states the initial concepts and defines the model considered. Loop flows and their link with multiple solutions to the power flow equations are discussed in Section III. Section IV gives a complete study of the multiple stable solutions to the power flow equations on a cycle network. Conclusions are given in Section $\square$

\section{DEFINITIONS AND FUNDAMENTAL CONCEPTS}

We are interested in a class of problems represented by at least three important physical systems. We have already defined the Kuramoto model in Eq. (1.1), for which more details can be found in review articles $\underline{\underline{4}-6}$. We have briefly mentioned vortices in superfluids and superconductors, as well as Josephson junction arrays, where circulating supercurrents are given by laws similar to Eq. (1.2) and for which a rather vast literature, including review articles, also exists $32-34$. These problems are well documented in the physics literature and we therefore do not discuss them further. Electric power grids are less known in physics and we start with a brief introduction to this third class of problems, emphasizing its connection with the Kuramoto model of Eq. (1.1). 


\section{A. Power Flow and Swing Equations}

Power grids are AC electric networks. They can be modeled as graphs with $n$ nodes where each node $i=1, \ldots, n$ injects (consumes) a power $P_{i}>0\left(P_{i}<0\right)$. The edges of the graph represent electrical lines with a complex admittance $Y=G+i B$. Power grids span different voltage levels separated by transformers which, to a good approximation, conserve power but neither current nor voltage. Additionally, the control variables are the injected and consumed powers, therefore the equations governing the behavior of the system are expressed in terms of electric powers and not currents. Considering a generating power plant, the balance between the source (mechanical, thermal, chemical or nuclear) power, the transmitted (electric) power and the losses leads to the swing equations 13

$$
\dot{\theta}_{i}=P_{i}-\sum_{j=1}^{n}\left|V_{i}\right|\left|V_{j}\right|\left[G_{i j} \cos \left(\theta_{i}-\theta_{j}\right)+B_{i j} \sin \left(\theta_{i}-\theta_{j}\right)\right], \quad i=1, \ldots n,
$$

where $\theta_{i}$ is the angle between the currents $I_{i}=\left|I_{i}\right| \exp (i \omega t)$ and the voltages $V_{i}=\left|V_{i}\right| \exp \left(i \omega t+i \theta_{i}\right)($ in a frame rotating with the frequency $\omega / 2 \pi=50$ or $60 \mathrm{~Hz}$ of the grid), $\theta_{i}-\theta_{j}$ is taken in $(-\pi, \pi]$ and $G$ and $B$ are the conductance and susceptance matrices respectively ${ }^{13}$. In Eq. (2.1), we already consider a simplified version of the swing equations, where we neglected the inertia of the (rotating) generators. We did that since our main interest is to determine whether a solution is stable or not, which is not influenced by the presence of an inertia term (note that the inertia influences stability time scales ${ }^{13}$ ). In most of our discussion we make a second approximation and consider networks of purely susceptive lines with $G_{i j}=0$. This is a leading order approximation in the small parameter $G_{i j} / B_{i j}<0.1$ valid for very high voltage networks. With this approximation, lines have no ohmic losses and all nodes are at the same voltage. For the sake of simplicity, we will also consider lines with identical capacities and set $K:=\left|V_{i}\right|\left|V_{j}\right| B_{i j}$, on all edges $\langle i j\rangle$. With all these approximations, Eq. (2.1) leads to an equation similar to Eq. (1.1),

$$
\dot{\theta}_{i}=P_{i}-K \sum_{j \sim i} \sin \left(\theta_{i}-\theta_{j}\right), \quad i=1, \ldots n
$$

where the sum is taken over nodes $j$ connected to node $i(j \sim i)$ and phase-locked solutions are governed by the power flow equations, which reduce to the form of Eq. (1.2),

$$
P_{i}=K \sum_{j \sim i} \sin \left(\theta_{i}-\theta_{j}\right), \quad i=1, \ldots n
$$

Electric power grids are balanced in steady-state, meaning that power injections exactly compensate power consumptions, i.e.

$$
\sum_{i} P_{i}=0
$$

Additionally, injected and consumed powers are confined to a compact support, $P_{i} \in\left[P_{\min }, P_{\max }\right]$ which is necessary for the existence of phase-locked synchronous solutions ${ }^{7,8}$.

We note finally that the quantity $P_{i j}:=K \sin \left(\theta_{i}-\theta_{j}\right)$ represents the power flow along line $\langle i j\rangle$, from site $i$ to site $j$, so that Eq. (2.3) can be rewritten as

$$
P_{i}=\sum_{j \sim i} P_{i j}
$$


which is Kirchhoff's currents law. Below we often use the power flow terminology and in particular we discuss loop flows to describe circulating flows around closed cycles that do not distribute power to consuming nodes. Eq. (2.3) only depends on angle differences, thus any solution is defined up to an homogeneous displacement of all angles. This gauge invariance allows to arbitrarily define a reference node whose angle is set to zero. All other angles are then determined with respect to that reference angle.

\section{B. Stability}

The swing equations, Eq. (2.2), govern the system's dynamics and allow to determine the linear stability of solutions of Eq. (2.3). Under small perturbations about such a phase-locked solution, $\theta_{i}^{(0)} \rightarrow \theta_{i}^{(0)}+\delta \theta_{i}$, the linearized dynamics reads

$$
\delta \dot{\theta}_{i}=-\sum_{j \sim i} K \cos \left(\theta_{i}^{(0)}-\theta_{j}^{(0)}\right)\left(\delta \theta_{i}-\delta \theta_{j}\right), \quad i=1, \ldots n
$$

The linear stability of the solution $\left\{\theta_{i}^{(0)}\right\}$ is therefore determined by the spectrum of the stability matrix $M\left(\left\{\theta_{i}^{(0)}\right\}\right)$,

$$
M_{i j}:= \begin{cases}K \cos \left(\theta_{i}^{(0)}-\theta_{j}^{(0)}\right), & \text { if } i \neq j, \\ -\sum_{k \sim i} K \cos \left(\theta_{i}^{(0)}-\theta_{k}^{(0)}\right), & \text { if } i=j,\end{cases}
$$

which depends on the angles at the phase-locked solution. The eigenvalues of $M\left(\left\{\theta_{i}^{(0)}\right\}\right)$ are called Lyapunov exponents. Because $\sum_{j} M_{j i}=\sum_{j} M_{i j}=0$, for all $i$, the constant vector is an eigenvector of $M$ with eigenvalue $\lambda_{1}=0$. This follows from the above mentioned gauge invariance, where only angle differences between oscillators matter. Furthermore, as $M$ is real symmetric, all its eigenvalues are real. Thus the synchronous state is stable if $M\left(\left\{\theta_{i}^{(0)}\right\}\right)$ is negative semidefinite and unstable otherwise. In other words, the synchronous solution remains stable as long as the largest nonvanishing eigenvalue $\lambda_{2}$ of $M\left(\left\{\theta_{i}^{(0)}\right\}\right)$ remains negative.

To the best of our knowledge, it was first mentioned in Ref. 35 that as long as all angle differences are in $[-\pi / 2, \pi / 2]$, Gershgorin's circle theorem ${ }^{36}$ guarantees that $M$ is negative semi-definite. Then all Lyapunov exponents are non-positive, which implies that any solution of Eq. (2.3) with $\theta_{i}^{(0)}-\theta_{j}^{(0)} \in$ $[-\pi / 2, \pi / 2]$ on each of the graph's edges is linearly stable. The same theorem allows to conclude that if $\left|\theta_{i}^{(0)}-\theta_{j}^{(0)}\right|>\pi / 2$ on all edges, the solution is linearly unstable. Recent works have investigated solutions with a single angle difference larger than $\pi / 2$ in a Kuramoto model on a cycle network ${ }^{29,30}$. However, little is known analytically if some of the angle differences are smaller and some are larger than $\pi / 2$, except on cycle networks with unidirectional nearest-neighbor coupling 27 . Below we fill this gap and show that at most one angle difference is bigger than $\pi / 2$ and that a stable solution with one angle difference exceeding $\pi / 2$ comes from a solution at larger $K$ with all angle differences smaller than $\pi / 2$. 


\section{LOOP FLOWS AND WINDING NUMBER}

In this section we show that different solutions of Eq. (2.3) for any network differ only by circulating loop flows. This rigorous result, which appeared in slightly different form in Ref. 37, sheds light on the common wisdom that Eq. (2.3) may have multiple stable solutions for networks with closed cycles $12,19,27,31$. Before we discuss this theorem, we recall some definitions from graph theory which we will use.

Definition III.1. A graph $G=\left(\mathcal{V}_{G}, E_{G}\right)$ is a set of vertices $\mathcal{V}_{G}$ with a set of edges $E_{G}$, each of which is a pair of connected vertices. If $i, j \in \mathcal{V}_{G}$, the edge connecting $i$ to $j$ is $\langle i j\rangle \in E_{G}$.

Definition III.2. A path from vertex $i$ to vertex $j$ in a graph $G$ is a sequence $S \subset E_{G}$ of edges

$$
S=\left\{\left\langle i i_{1}\right\rangle,\left\langle i_{1} i_{2}\right\rangle, \ldots,\left\langle i_{\ell} j\right\rangle\right\}
$$

Definition III.3. A graph is connected if for any two vertices $i, j \in \mathcal{V}_{G}$ there exists a path from $i$ to $j$.

Definition III.4. A cycle in a graph is a path from a vertex $i$ to itself going at most once through any edge.

Definition III.5. A tree is a connected graph with no cycle. Given a graph $G=\left(\mathcal{V}_{G}, E_{G}\right)$, a spanning tree $T$ of $G$ is a tree such that $\mathcal{V}_{T}=\mathcal{V}_{G}$ and $E_{T} \subset E_{G}$.

Remarks. (i) It can be shown inductively that a tree with $n$ vertices has exactly $n-1$ edges.

(ii) On a tree-network, there is a unique flow distribution satisfying Kirchhoff's current law.

In what follows, we use the terms network and grid to denote physical objects, whose mathematical representations will be referred to as graphs. Additionally, we introduce the concept of loop flows, which are constant power flows circulating clockwise or anticlockwise around a cycle in a network. Strictly speaking, loop flows can be univocally defined only when power is neither injected nor consumed in the network. With finite power injections and consumptions, loop flows can be defined only relatively, as flow differences from a reference solution, in the spirit of the upcoming theorem.

Let $G$ be a graph and $O_{G}$ an arbitrary orientation of this graph, which means that we define positive and negative directions for every edge of $G$ in the following way. For each edge $\langle i j\rangle$ we call the vertex $i$ the source of the edge and $j$ its target. Consider the real vector space $\mathcal{I} \simeq \mathbb{R}^{m}$ of flows on the $m$ edges of a graph $G$. The components $\left\{I_{\ell}\right\}$ of a flow vector $\boldsymbol{I} \in \mathcal{I}$ describe the intensity of the flow on the $\ell^{\text {th }}$ edge of $G$, with $I_{\ell}>0$ if the direction of the flow agrees with the orientation of this edge given by $O_{G}$, and $I_{\ell}<0$ otherwise. The canonical basis of $\mathcal{I}$ is the set of flow vectors $\boldsymbol{J}_{\ell}, \ell=1, \ldots, m$, with unit flow on edge $\ell$ and zero flow on all other edges. Given a vector of power injections and consumptions at every node,

$$
\boldsymbol{P}=\left(P_{1}, \ldots, P_{n}\right) \in(1, \ldots, 1)^{\perp} \subset \mathbb{R}^{n}
$$

a flow vector $I \in \mathcal{I}$ satisfies Kirchhoff's current law $P_{i}=\sum_{j \sim i} P_{i j}$ if

$$
P_{i}=\sum_{\ell} A_{i \ell} I_{\ell} \quad i=1, \ldots, n
$$


where we introduced the incidence matrix $A$ of $G$,

$$
A_{i \ell}:= \begin{cases}1, & \text { if node } i \text { is the source of edge } \ell \\ -1 & \text { if node } i \text { is the target of edge } \ell \\ 0, & \text { otherwise }\end{cases}
$$

We are now ready to formulate and prove our theorem.

Theorem III.6. Let $G=\left(\mathcal{V}_{G}, E_{G}\right)$ be a connected graph with $\left|\mathcal{V}_{G}\right|=n$ sites and $\left|E_{G}\right|=m$ edges. Let $\boldsymbol{P} \in(1, \ldots, 1)^{\perp}$ be a vector of power injections and consumptions at each node. Then two distributions of flows on $G$ represented by flow vectors $\boldsymbol{I}^{\prime}$ and $\boldsymbol{I}^{\prime \prime} \in \mathcal{I}$ satisfying Kirchhoff's currents law, Eq. (2.4), differ by a combination of loop flows on the different cycles of $G$.

Remark. In particular, Theorem III.6 implies that the flow distributions of two different solutions of Eq. (2.3) differ by a collection of loop flows. This result already appeared in slightly different form in the Supporting Information of Ref. 3\%.

Proof. If $m=n-1$, then $G$ is a tree and the flows on the lines are uniquely determined, which agrees with the statement because $G$ has no cycle. Therefore, from now on we assume $m \geq n$. Let $T$ be a spanning tree of $G$ and let us number the edges of $T$ from 1 to $n-1$ and the edges of $G \backslash T$ from $n$ to $m$. Let $\boldsymbol{I}^{\circ}:=\boldsymbol{I}^{\prime}-\boldsymbol{I}^{\prime \prime}$ be the difference between the two flow vectors. Then, for any $i$ we have

$$
\sum_{\ell} A_{i \ell} I_{\ell}^{\circ}=\sum_{\ell} A_{i \ell}\left(I_{\ell}^{\prime}-I_{\ell}^{\prime \prime}\right)=P_{i}-P_{i}=0
$$

from which we conclude that $\boldsymbol{I}^{\circ}$ is a solution of Eq. (3.1) with $\boldsymbol{P}=0$. What we need to show is therefore that any solution $\boldsymbol{I}$ of the system of equations

$$
\sum_{\ell} A_{i \ell} I_{\ell}=0, \quad i=1, \ldots, n,
$$

is a combination of loop flows. To do this we write Eq. (3.2) in matricial form,

$$
A \boldsymbol{I}=0 .
$$

By definition, the set of solutions of Eq. (3.3) is the kernel of $A$, which is a subspace of $\mathcal{I}$.

In algebraic graph theory, $\operatorname{ker}(A)$ is referred to as the cycle space and it is a standard result 38 that any element in $\operatorname{ker}(A)$ is a linear combination of unitary flows along the cycles of the network considered. This completes the proof.

Remark. Theorem III.6 is not restricted to the power flow problem. It generically applies to any system of coupled oscillators with antisymmetric coupling, in particular to the Kuramoto model on any network.

Indexing the nodes along one such cycle, we write $P_{i, i+1}$ for the power flow from node $i$ to node $i+1$, with indices taken modulo $n$. Theorem III.6 states in particular that multiple solutions to Eq. (2.3) can appear only when there are closed cycles in the network. 
Alternatively, any flow $\left\{P_{i, i+1}\right\}$ on a cycle can be written as the sum of a reference solution, characterized by its flows $\left\{P_{i, i+1}^{*}\right\}$, and a loop flow of intensity $K \varepsilon$, circulating around the cycle (see Fig. 11),

$$
P_{i, i+1}=P_{i, i+1}^{*}+K \varepsilon .
$$

We call $\varepsilon \in[-1,1]$ the loop flow parameter. It is only defined with respect to a reference solution, which is conveniently constructed from the $P_{i}$ 's as

$$
P_{i, i+1}^{*}:=\sum_{j=1}^{i} P_{j}, \quad i=1, \ldots, n .
$$

Note that the reference solution depends on node numbering and any other flow distribution satisfying Kirchhoff's power balance can be taken as reference solution.

As angle differences $\Delta_{i j}:=\theta_{i}-\theta_{j}$ are taken modulo $2 \pi$ in the interval $(-\pi, \pi]$, their sum over the cycle gives an integer multiple of $2 \pi$. This brings us to the definition of the winding number.

Definition III.7. For a given solution of the power flow Eq. (2.3) on the cycle, we define its winding number as the integer

$$
q:=(2 \pi)^{-1} \sum_{i=1}^{n} \Delta_{i, i+1} \in \mathbb{Z}
$$

The winding number characterizes a solution and is related to the loop flow. Unlike the latter, however, it is uniquely defined. Eq. (3.4) quantizes the loop flow, i.e. it can take only discrete values.

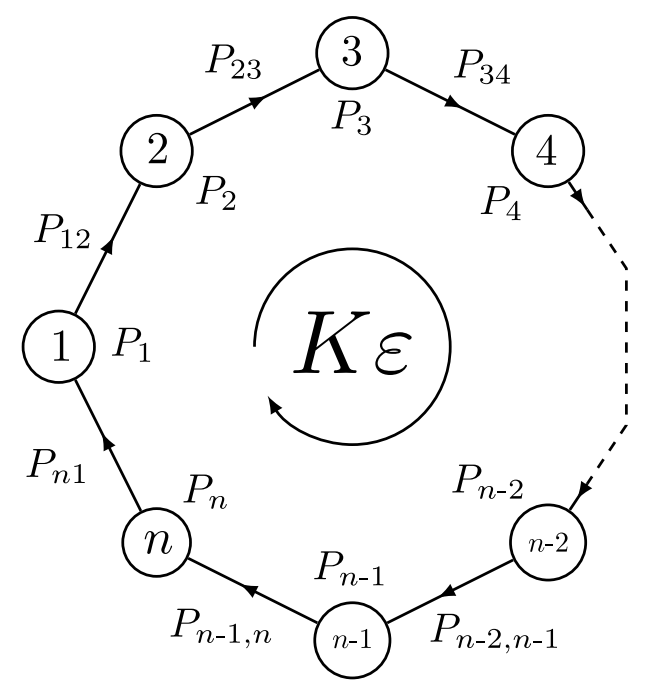

FIG. 1. Cycle network of length $n$. The nodes inject/consume a power $P_{i}$, while a power $P_{i j}$ is transmitted along the edge $\langle i j\rangle$. Edges correspond to lines with capacity $K$ (loop flow problem), to couplings of strength $K$ (Kuramoto problem) or to tunnel barriers of transparency $\hbar K / 8 e_{s}$ (Josephson junction problem). A loop flow of intensity $K \varepsilon$ is circulating around the cycle, where $\varepsilon \in[-1,1]$. 


\section{THE NUMBER OF STABLE SOLUTIONS}

Theorem III.6 connects the existence of multiple solutions to Eq. 2.3) to the presence of cycles traveled by quantized loop flows. The number of solutions is thus related to the number of acceptable, discrete loop flows. In the remainder of this manuscript, we focus on this problem in single-cycle graphs.

We first treat the case $K \rightarrow \infty$, where stable solutions necessarily have all angle differences in $[-\pi / 2, \pi / 2]$. We then consider the situation for finite $K$, where we show that the number of stable solutions decreases with $K$, that the angle difference along some of the lines can exceed $\pi / 2$, but that it can happen on a single line at most.

\section{A. Angle differences and sum of angle differences}

A solution is fully characterized by the angle differences along the lines. These can be written as functions of the loop flow parameter $\varepsilon$,

$$
P_{i, i+1}=P_{i, i+1}^{*}+K \varepsilon=K \sin \left(\Delta_{i, i+1}\right) \quad \Longrightarrow \quad \Delta_{i, i+1}=a_{i}(\varepsilon),
$$

where there are two possible choices for each $a_{i}$,

$$
a_{i}(\varepsilon)=\left\{\begin{array}{l}
\arcsin \left(\varepsilon+P_{i, i+1}^{*} / K\right) \\
\pi-\arcsin \left(\varepsilon+P_{i, i+1}^{*} / K\right) \quad \Longrightarrow \Delta_{i, i+1} \in[-\pi / 2, \pi / 2] \\
\Delta_{i, i+1} \in(-\pi,-\pi / 2) \cup(\pi / 2, \pi]
\end{array}\right.
$$

Since the power transmitted along any link is bounded by $K$, we obtain bounds on $\varepsilon$,

$$
-K \leq P_{i, i+1} \leq K \Longleftrightarrow-1-P_{i, i+1}^{*} / K \leq \varepsilon \leq 1-P_{i, i+1}^{*} / K, \quad i=1, \ldots, n .
$$

Thus $\varepsilon \in\left[\varepsilon_{\min }, \varepsilon_{\max }\right]$, with

$$
\varepsilon_{\min }:=\max _{1 \leq i \leq n}\left\{-1-P_{i, i+1}^{*} / K\right\}=-1-P_{\min }^{*} / K
$$

and

$$
\varepsilon_{\max }:=\min _{1 \leq i \leq n}\left\{1-P_{i, i+1}^{*} / K\right\}=1-P_{\max }^{*} / K
$$

where $P_{\min }^{*}:=\min _{i} P_{i, i+1}^{*}$ and $P_{\max }^{*}:=\max _{i} P_{i, i+1}^{*}$. Note that as soon as the $P_{i}$ 's are not all equal to zero, $P_{\min }^{*} \neq P_{\max }^{*}$. We add an appropriate constant to the reference flow to make sure that $P_{\min }^{*} \neq 0$ and $P_{\max }^{*} \neq 0$, which will facilitate our discussion without restricting its generality.

As seen in Section IB, a solution is stable if all angle differences belong to the interval $[-\pi / 2, \pi / 2]$. In this situation, we can write the sum of angle differences around the cycle as a function of the parameter $\varepsilon$,

$$
\mathcal{A}_{0}(K, \varepsilon):=\sum_{i=1}^{n} \Delta_{i, i+1}=\sum_{i=1}^{n} \arcsin \left(\varepsilon+P_{i, i+1}^{*} / K\right) .
$$

As the arcsine is continuous and increasing, the function $\mathcal{A}_{0}$ is also continuous and increasing with respect to $\varepsilon$. Thus for fixed $K_{0}$, the function $\mathcal{A}_{0}\left(K_{0}, \varepsilon\right)$ defines a one-to-one correspondence between the intervals

$$
\left[\varepsilon_{\min }\left(K_{0}\right), \varepsilon_{\max }\left(K_{0}\right)\right] \longleftrightarrow\left[\mathcal{A}_{0}\left(K_{0}, \varepsilon_{\min }\left(K_{0}\right)\right), \mathcal{A}_{0}\left(K_{0}, \varepsilon_{\max }\left(K_{0}\right)\right)\right]
$$


The sum of angle differences around the cycle has to be a multiple of $2 \pi$, thus defining $\varepsilon_{q}$ such that $\mathcal{A}_{0}\left(K_{0}, \varepsilon_{q}\right)=2 \pi q$ and

$$
\Delta_{i, i+1}=\arcsin \left(\varepsilon_{q}+P_{i, i+1}^{*} / K_{0}\right), \quad i=1, \ldots, N,
$$

gives a stable solution of Eq. (2.3).

Therefore, the number of solutions with $\left|\Delta_{i, i+1}\right|<\pi / 2$ for all $i$ is straightforwardly given by the number of $q$ 's such that $\mathcal{A}_{0}\left(K_{0}, \varepsilon_{q}\right)=2 \pi q$. Previous works have treated this case ${ }^{28}$, however allowing $\left|\Delta_{i, i+1}\right|>\pi / 2$ renders the problem much more complicated. It has so far been solved only for unidirectional coupling 27 . Our strategy for incorporating solutions with $\left|\Delta_{i, i+1}\right|>\pi / 2$ is to first treat $K \rightarrow \infty$, where we show that $\left|\Delta_{i, i+1}\right|<\pi / 2$, for all $i$, for stable solutions. The number of solutions is then easy to compute. Second, we generalize the study to finite $K$ and see that the number of solutions obtained for $K \rightarrow \infty$ is an upper bound on the number of solutions for any finite $K$.

\section{B. Infinite capacity}

The case $K \rightarrow \infty$ is equivalent to the identical oscillators case with $P_{i}=0$, for all $i$. In this limit, the bounds on $\varepsilon$ are

$$
\lim _{K \rightarrow \infty} \varepsilon_{\max }(K)=1, \quad \lim _{K \rightarrow \infty} \varepsilon_{\min }(K)=-1,
$$

and thus

$$
\lim _{K \rightarrow \infty} \mathcal{A}_{0}\left(K, \varepsilon_{\min }(K)\right)=-n \pi / 2, \quad \quad \quad \lim _{K \rightarrow \infty} \mathcal{A}_{0}\left(K, \varepsilon_{\max }(K)\right)=n \pi / 2
$$

An $\varepsilon_{q}$ is associated to each integer multiple of $2 \pi$ in $[-n \pi / 2, n \pi / 2]$ corresponding to a stable solution of Eq. (2.3). There are $\mathcal{N}=2 \operatorname{Int}[n / 4]+1$ such integers. This is illustrated in Fig. 2,

Theorem IV.1. For $K \rightarrow \infty$, any stable solution of the power flow Eq. (2.3) on a cycle network has all angle differences in $[-\pi / 2, \pi / 2]$. Furthermore all angle differences are equal to $2 \pi q / n$, where $q$ is the winding number of the solution.

Remarks. (i) The principal minors of a matrix $A$ are the determinants of the square submatrices of $A$ with the same row and column indices. Sylvester's criterion states that a matrix is positive semi-definite if and only if all its principal minors are non-negative $\underline{36}$.

(ii) The result of Theorem IV.1 was already known for unidirectional coupling 27 . Here we extend this result to bidirectional interactions. Furthermore, our approach allows to relate the finite $K$ situation to the infinite $K$ situation.

Proof. The power flow along line $\langle i, i+1\rangle$ is

$$
P_{i, i+1}=K \sin \left(\Delta_{i, i+1}\right)=P_{i, i+1}^{*}+K \varepsilon,
$$

where $\left\{P_{i, i+1}^{*}\right\}$ is a reference solution constructed from finite powers. Thus when $K \rightarrow \infty$, the sine of the angle difference along every line of the cycle tends to the same value,

$$
\lim _{K \rightarrow \infty} \sin \left(\Delta_{i, i+1}\right)=\varepsilon
$$


This implies that the angle difference along each line of the network belongs to the set $\{\arcsin (\varepsilon), \pi-$ $\arcsin (\varepsilon)\}$ and thus the cosine of the angle differences along all the lines takes the same absolute value with either positive or negative sign. First of all, if all angle differences $\operatorname{are} \arcsin (\varepsilon)$, the stability matrix defined in Eq. (2.6) is easily expressed as

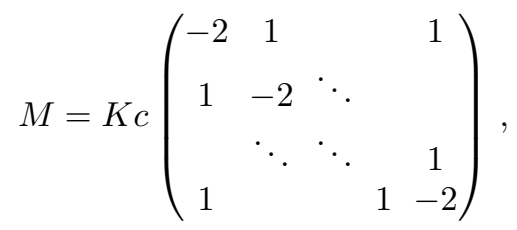

where $c:=\cos (\arcsin (\varepsilon))=\sqrt{1-\varepsilon^{2}}$. This matrix is negative semi-definite and has only non-positive eigenvalues by Gershgorin's circle theorem ${ }^{36}$. Thus the solution is stable. Now, if all angle differences are $\pi-\arcsin (\varepsilon)$, then all cosines are negative and the stability matrix is obviously positive semidefinite. The solution is then unstable. Let us now consider the mixed case where at least one angle difference is $\arcsin (\varepsilon)$ and one is $\pi-\arcsin (\varepsilon)$. In this case, there exists at least one node $i$ such that $\Delta_{i-1, i}=\arcsin (\varepsilon)$ and $\Delta_{i, i+1}=\pi-\arcsin (\varepsilon)$ and the corresponding stability matrix has the form

$$
M^{\prime}=K c\left(\begin{array}{ccccc}
\ddots & \ddots & & & \\
\ddots & x & 1 & 0 & \\
& 1 & 0 & -1 & \\
& 0 & -1 & y & \ddots \\
& & & \ddots & \ddots
\end{array}\right) .
$$

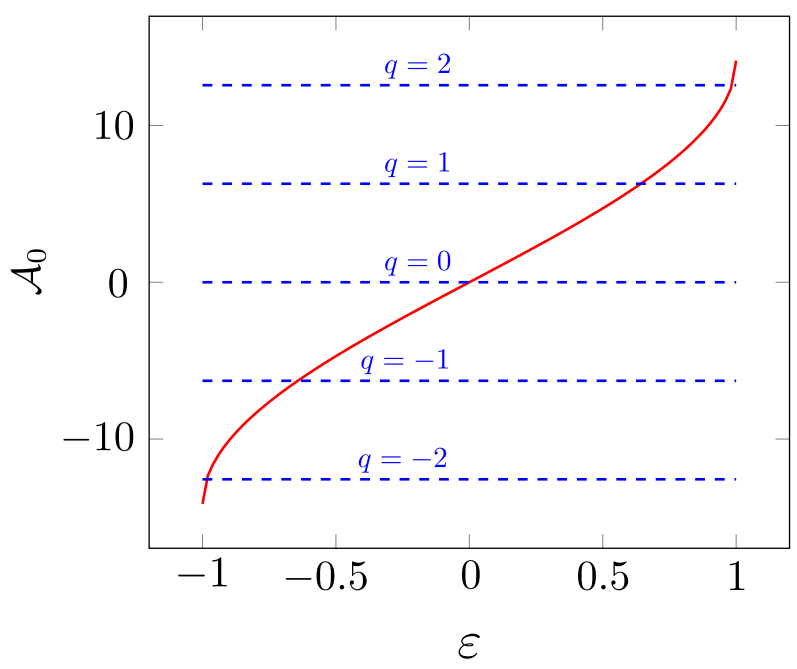

FIG. 2. Plot of $\mathcal{A}_{0}(K, \varepsilon)$ as a function of $\varepsilon$ (red), for a cycle network of length $n=9$, with $K \rightarrow \infty$. Horizontal dashed lines correspond to $\mathcal{A}_{0}=2 \pi q$ with $q$ values indicated. Each intersection of the red line with a blue dashed line gives a stable solution of Eq. (2.3). 
The principal minor of $-M^{\prime}$ with row and column indices $\{i, i+1\}$ is

$$
\left|\begin{array}{cc}
0 & +1 \\
+1 & -y
\end{array}\right|=-1
$$

which, by Sylvester's criterion ${ }^{36}$, implies that $M^{\prime}$ is not negative semi-definite. In other words, $M^{\prime}$ has at least one positive eigenvalue and thus the solution is unstable. From this we conclude that the stable solutions for sufficiently large $K$ all have angle differences in $[-\pi / 2, \pi / 2]$. They are captured by finding the intersections of $\mathcal{A}_{0}(K, \varepsilon)$ with integer multiples of $2 \pi$ as illustrated in Fig. 2 .

Let $q$ be the winding number of a stable solution for $K \rightarrow \infty$. As all angle differences have the same value $\Delta \in[-\pi / 2, \pi / 2]$, we have

$$
2 \pi q=\sum_{i=1}^{n} \Delta_{i, i+1}=n \Delta \Longrightarrow \Delta=2 \pi q / n
$$

The corresponding angles are $\theta_{i}=-2 \pi q i / n$, taken in the interval $(-\pi, \pi]$.

\section{Finite capacity}

We now consider finite values for $K$ and $P_{i}$ 's not all equal to zero. We first show that the number of solutions to the power flow Eq. (2.3) with all angle differences in $[-\pi / 2, \pi / 2]$ decreases with $K$. Second, we show that for finite $K$, there exist stable solutions with one angle difference in $(-\pi,-\pi / 2) \cup(\pi / 2, \pi]$, and we relate them to solutions at larger $K$ with all angle differences in $[-\pi / 2, \pi / 2]$. This gives an analytical confirmation of the numerical observations of Tilles et al. $\stackrel{29}{ }$, and of Roy and Lahiri ${ }^{30}$.

Proposition IV.2. For a one-cycle network with $n$ nodes, if $K$ decreases, then $\mathcal{A}_{0}\left(K, \varepsilon_{\min }\right)$ increases and $\mathcal{A}_{0}\left(K, \varepsilon_{\max }\right)$ decreases.

Proof. From Eqs. (4.3b) and (4.4) the derivative of $\mathcal{A}_{0}$ with respect to $K$ reads

$$
\frac{d \mathcal{A}_{0}\left(K, \varepsilon_{\mathrm{min}}\right)}{d K}=\sum^{\prime}\left[1-\left(-1+\frac{P_{i, i+1}^{*}-P_{\min }^{*}}{K}\right)^{2}\right]^{-\frac{1}{2}} \frac{P_{\min }^{*}-P_{i, i+1}^{*}}{K^{2}},
$$

where $\Sigma^{\prime}$ indicates that the sum is taken over indices $j$ such that $P_{j, j+1}^{*}>P_{\min }^{*}$. This sum is obviously non-positive. In the same way it is easily seen that

$$
\frac{d \mathcal{A}_{0}\left(K, \varepsilon_{\max }\right)}{d K} \geq 0
$$

Proposition IV.2 implies that as $K$ decreases, the interval of values of $\mathcal{A}_{0}$ gets smaller and contains fewer and fewer multiples of $2 \pi$. We show now that for finite capacities, the stable solutions are directly related to the stable solutions for $K \rightarrow \infty$, even if some of them have angle differences in $(-\pi,-\pi / 2) \cup(\pi / 2, \pi]$. First we define

$$
\mathcal{A}_{j}(K, \varepsilon):=\sum_{i \neq j} \arcsin \left(\varepsilon+P_{i, i+1}^{*} / K\right)+\pi-\arcsin \left(\varepsilon+P_{j, j+1}^{*} / K\right), \quad j=1, \ldots, n .
$$


The function $\mathcal{A}_{0}$ is the sum of angle differences all taken in the interval $[-\pi / 2, \pi / 2]$ and for $j=1, \ldots, n$, the function $\mathcal{A}_{j}$ is this sum with one angle difference, the $j^{\text {th }}$, taken in $(-\pi,-\pi / 2) \cup(\pi / 2, \pi]$. We also introduce the following notation

$$
c_{i}:=\cos \left(\Delta_{i, i+1}\right) .
$$

The sign of $c_{i}$ depends on our choice for $\Delta_{i, i+1}$,

$$
c_{i}=\left\{\begin{array}{c}
\cos \left[\arcsin \left(\varepsilon+P_{i, i+1}^{*} / K\right)\right]=\sqrt{1-\left(\varepsilon+P_{i, i+1}^{*} / K\right)^{2}}, \\
\cos \left[\pi-\arcsin \left(\varepsilon+P_{i, i+1}^{*} / K\right)\right]=-\sqrt{1-\left(\varepsilon+P_{i, i+1}^{*} / K\right)^{2}} .
\end{array}\right.
$$

The domain $\mathcal{D}$, in the $(K, \varepsilon)$-plane, where the functions $\mathcal{A}_{j}$ are defined, is such that each arcsine is well-defined,

$$
\mathcal{D}=\left\{(K, \varepsilon): \varepsilon+P_{\min }^{*} / K \geq-1, \varepsilon+P_{\max }^{*} / K \leq 1\right\} .
$$

By definition, in the interior of $\mathcal{D}$, the $c_{i}$ 's are nonzero. Let us define the upper and lower boundaries of $\mathcal{D}$,

$$
\begin{aligned}
& \mathcal{D}_{1}:=\left\{(K, \varepsilon): \varepsilon+P_{\max }^{*} / K=1\right\}, \\
& \mathcal{D}_{0}:=\left\{(K, \varepsilon): \varepsilon+P_{\min }^{*} / K=-1\right\} .
\end{aligned}
$$

We next denote by $j_{0}$ and $j_{1}$ the indices such that $P_{j_{0}, j_{0}+1}^{*}=P_{\min }^{*}$ and $P_{j_{1}, j_{1}+1}^{*}=P_{\max }^{*}$ respectively. Note that for $(K, \varepsilon) \in \mathcal{D}_{1}\left[\right.$ resp. $\left.(K, \varepsilon) \in \mathcal{D}_{0}\right]$, the functions $\mathcal{A}_{0}(K, \varepsilon)$ and $\mathcal{A}_{j_{1}}(K, \varepsilon)$ [resp. $\left.\mathcal{A}_{j_{0}}(K, \varepsilon)\right]$ have the same value.

Remark. It is possible that multiple lines carry the same maximal or minimal power. In this case these indices are not uniquely defined, but we are free to choose any $j_{0}$ and $j_{1}$ satisfying $P_{j_{0}, j_{0}+1}^{*}=P_{\min }^{*}$ and $P_{j_{1}, j_{1}+1}^{*}=P_{\max }^{*}$.

For any choice of $a_{i}$ 's in Eq. (4.2), any point $(K, \varepsilon) \in \mathcal{D}$ such that $\sum_{i} a_{i}=2 \pi q$ is a solution (not necessarily stable) of Eq. (2.3). Hence we now study the $2 \pi q$-level sets of $\mathcal{A}_{j}$, for $q \in \mathbb{Z}$ and $j=0, \ldots, n$. Note first that as $\mathcal{A}_{j}$ is smooth in the interior of the domain $\mathcal{D}$ for any $j$, the Implicit Function Theorem ${ }^{39}$ implies that its level sets are level curves. For any $K_{0}$, we call $\mathcal{S}\left(K_{0}\right) \subset\left[\varepsilon_{\min }, \varepsilon_{\max }\right]$ the set of $\varepsilon$-values corresponding to stable solutions of the power flow Eq. (2.3), i.e. such that there exists a choice of $\left\{a_{i}\right\}$ in Eq. (4.2) for which

$$
\sum_{i} a_{i}\left(K_{0}, \varepsilon\right)=2 \pi q, \quad q \in \mathbb{Z} .
$$

Let $\mathcal{N}\left(K_{0}\right):=\left|\mathcal{S}\left(K_{0}\right)\right|$ be its cardinality. The main results of this section are the following theorem on the properties of $\mathcal{N}(K)$ and its corollary.

Theorem IV.3. The number of stable solutions of the power flow equations, $\mathcal{N}(K)$ is a monotonically increasing function of $K$.

Corollary IV.4. The value

$$
\mathcal{N}_{\infty}:=\lim _{K \rightarrow \infty} \mathcal{N}(K)=2 \operatorname{Int}[n / 4]+1,
$$

is an upper bound on the number of stable solutions of Eq. (2.3) on a cycle network, independently of $K$ and $\left\{P_{i}\right\}$. 
The proof of Theorem IV.3 relies on five lemmas. A major ingredient of the proof is that the functions $\mathcal{A}_{j}(K, \varepsilon)$, for $j=1, \ldots, n$, have no critical points. This fact and Lemma IV.7 give precise informations about the shape of the level curves of $\mathcal{A}_{j}$.

Lemma IV.5. For $j \in\{1, \ldots, n\}$, the function $\mathcal{A}_{j}$ has no critical point in the interior of $\mathcal{D}$.

Proof. For $j \in\{1, \ldots, n\}$, we have

$$
\frac{\partial \mathcal{A}_{j}}{\partial \varepsilon}=\sum_{k} c_{k}^{-1}
$$

Assume first that at some point $(K, \varepsilon)$ in the interior of $\mathcal{D}, \partial \mathcal{A}_{j} / \partial \varepsilon=0$, then

$$
\frac{\partial \mathcal{A}_{j}}{\partial \varepsilon}=0 \Longleftrightarrow \sum_{k} c_{k}^{-1}=0 \Longleftrightarrow \sum_{k \neq j} c_{k}^{-1}=-c_{j}^{-1} \Longleftrightarrow \sum_{k \neq j}-c_{j} / c_{k}=1 .
$$

Recall that as we chose

$$
\Delta_{j, j+1}=\pi-\arcsin \left(\varepsilon+P_{j, j+1}^{*} / K\right),
$$

we have $c_{j}<0$. It is then easy to check that for any $k \neq j$

$$
\begin{aligned}
0<-c_{j} / c_{k}<1 & \Longrightarrow 0<-c_{j}<c_{k} \\
& \Longrightarrow \sqrt{1-\left(\varepsilon+P_{j, j+1}^{*} / K\right)^{2}}<\sqrt{1-\left(\varepsilon+P_{k, k+1}^{*} / K\right)^{2}} \\
& \Longrightarrow\left(\varepsilon+P_{j, j+1}^{*} / K\right)^{2}>\left(\varepsilon+P_{k, k+1}^{*} / K\right)^{2} .
\end{aligned}
$$

There are now two possible cases :

1. if $\varepsilon+P_{j, j+1}^{*} / K>0$, then

$$
\begin{aligned}
\varepsilon+P_{j, j+1}^{*} / K>\varepsilon+P_{k, k+1}^{*} / K & \Longleftrightarrow P_{j, j+1}^{*}>P_{k, k+1}^{*}, \quad \forall k \neq j \\
& \Longrightarrow P_{j, j+1}^{*}=P_{\max }^{*} ;
\end{aligned}
$$

2. if $\varepsilon+P_{j, j+1}^{*} / K<0$, then

$$
\begin{aligned}
\varepsilon+P_{j, j+1}^{*} / K<\varepsilon+P_{k, k+1}^{*} / K & \Longleftrightarrow P_{j, j+1}^{*}<P_{k, k+1}^{*}, \quad \forall k \neq j \\
& \Longrightarrow P_{j, j+1}^{*}=P_{\min }^{*}
\end{aligned}
$$

Thus if $j \notin\left\{j_{0}, j_{1}\right\}$, Eq. (4.8) cannot hold and $\mathcal{A}_{j}$ has no critical point in $\mathcal{D}$. Let now $j \in\left\{j_{0}, j_{1}\right\}$ and assume that

$$
\frac{\partial \mathcal{A}_{j}}{\partial \varepsilon}=\sum_{k} c_{k}^{-1}=0
$$


We calculate

$$
\begin{aligned}
\frac{\partial \mathcal{A}_{j}}{\partial K} & =\sum_{k \neq j} \frac{\partial}{\partial K} \arcsin \left(\varepsilon+P_{k, k+1}^{*} / K\right)+\frac{\partial}{\partial K}\left[\pi-\arcsin \left(\varepsilon+P_{j, j+1}^{*} / K\right)\right] \\
& =-\sum_{k \neq j} c_{k}^{-1} P_{k, k+1}^{*} / K^{2}-c_{j}^{-1} P_{j, j+1}^{*} / K^{2} \\
& =-\sum_{k \neq j} c_{k}^{-1} P_{k, k+1}^{*} / K^{2}+\sum_{k \neq j} c_{k}^{-1} P_{j, j+1}^{*} / K^{2} \\
& =\sum_{k \neq j}\left(P_{j, j+1}^{*}-P_{k, k+1}^{*}\right) /\left(K^{2} c_{k}\right),
\end{aligned}
$$

which is non zero as every term is non-negative (resp. non-positive) if $j=j_{1}$ (resp. $j=j_{0}$ ). Thus the partial derivatives of $\mathcal{A}_{j}$ are never simultaneously zero implying that $\mathcal{A}_{j}$ has no critical point in the domain $\mathcal{D}$.

Corollary IV.6. For any $j \in\{1, \ldots, n\}$, the level sets of $\mathcal{A}_{j}$ are continuous lines that: i) cannot end in the interior of $\mathcal{D}$, ii) are not closed and iii) have no trifurcation.

Proof. Any of these situations would imply at least one critical point.

Lemma IV.7. Let $L \in \mathbb{R}$. If there exist $K_{0} \in \mathbb{R}$ such that $\mathcal{A}_{j_{1}}\left(K_{0}, \varepsilon_{\max }\left(K_{0}\right)\right)=L$, then there is a single level curve of $\mathcal{A}_{j_{1}}=L$ starting at $\left(K_{0}, \varepsilon_{\max }\left(K_{0}\right)\right)$. The same holds for level curves of $\mathcal{A}_{j_{0}}=L$ starting at $\left(K_{0}, \varepsilon_{\min }\left(K_{0}\right)\right)$.

Remark. This lemma means that the red curve in Fig. 3 is unique.

Proof. We prove the first statement, the proof of the second one being similar. We first recall that

$$
\frac{\partial \mathcal{A}_{j_{1}}}{\partial K}=\left[1-\left(\varepsilon+\frac{P_{\max }^{*}}{K}\right)^{2}\right]^{-\frac{1}{2}} \frac{P_{\max }^{*}}{K^{2}}-\sum_{k \neq j_{1}}\left[1-\left(\varepsilon+\frac{P_{k, k+1}^{*}}{K}\right)^{2}\right]^{-\frac{1}{2}} \frac{P_{k, k+1}^{*}}{K^{2}} .
$$

Consider now a small interval around $K_{0}, I_{0}=\left[K_{0}-\delta_{0}, K_{0}+\delta_{0}\right]$. For any $K \in I_{0}$, there is a $\xi_{K}>0$ such that for all $\varepsilon \in\left(\varepsilon_{\max }(K)-\xi_{K}, \varepsilon_{\max }(K)\right)$, the first term in the right-hand-side of Eq. (4.9) dominates, and the partial derivative $\partial \mathcal{A}_{j_{1}} / \partial K$ has the same sign as $P_{\max }^{*}$, which we chose non-zero above (see discussion below Eq. (4.3b)). Thus setting

$$
\xi^{*}:=\min _{K \in I_{0}}\left\{\xi_{K}\right\}
$$

allows us to define a neighborhood $U \subset \mathcal{D}$ of $\left(K_{0}, \varepsilon_{\max }\left(K_{0}\right)\right)$,

$$
U:=\left\{(K, \varepsilon):\left|K-K_{0}\right|<\delta_{0}, 0 \leq \varepsilon_{\max }(K)-\varepsilon<\xi^{*}\right\},
$$

where the partial derivative $\partial \mathcal{A}_{j_{1}} / \partial K$ always has the same non-zero sign. This neighborhood is sketched in Fig. 3. But if there is more than one level curve starting at $\left(K_{0}, \varepsilon_{\max }\left(K_{0}\right)\right)$ and since $\mathcal{A}_{j_{1}}$ is not constant, this partial derivative has to change sign in any neighborhood of $\left(K_{0}, \varepsilon_{\max }\left(K_{0}\right)\right)$, which leads to a contradiction. There is therefore at most one such level curve. 
We next investigate how the linear stability of the solutions to the power flow Eq. (2.3) varies along the level curves $\mathcal{A}_{j}=2 \pi q$. The two following lemmas show that the only functions leading to stable solutions are $\mathcal{A}_{0}, \mathcal{A}_{j_{0}}$ and $\mathcal{A}_{j_{1}}$.

Lemma IV.8. For any choice of $a_{i}$ 's, the stability matrix $M$ has a second null eigenvalue $\lambda_{2}$ (see Section [IB $)$ if and only if

$$
\sum_{k} c_{k}^{-1}=0
$$

Proof. Consider the charateristic polynomial of the stability matrix $M$,

$$
\chi(M)=\left|\begin{array}{cccccc}
-c_{1}-c_{n}-\lambda & c_{1} & \cdots & 0 & \cdots & c_{n} \\
c_{1} & -c_{1}-c_{2}-\lambda & c_{2} & & & \vdots \\
\vdots & c_{2} & \ddots & \ddots & & 0 \\
0 & & \ddots & & & \vdots \\
\vdots & & & & & c_{n-1} \\
c_{n} & \cdots & 0 & \cdots & c_{n-1}-c_{n-1}-c_{n}-\lambda
\end{array}\right| .
$$

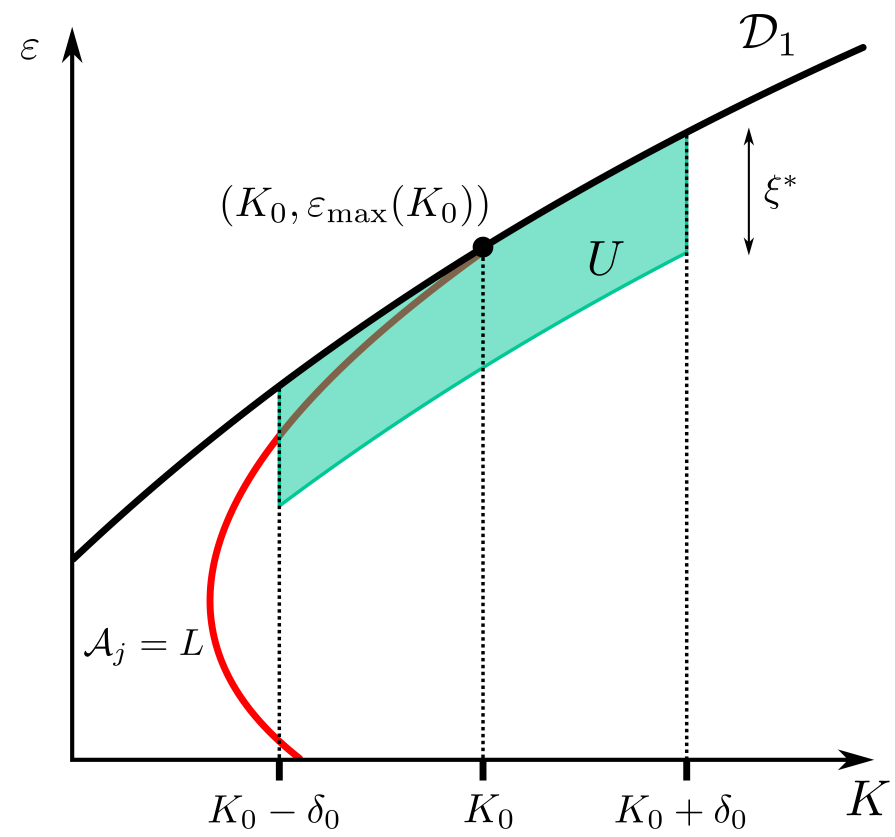

FIG. 3. Sketch of the level curve $\mathcal{A}_{j}=0$ (red curve) and of the neighborhood $U$ of $\left(K_{0}, \varepsilon_{\max }\left(K_{0}\right)\right)$ where the partial derivative of $\mathcal{A}_{j}$ with respect to $K$ always has the same non-zero sign. The neighborhood $U$ is bounded above by the boundary of the domain $\mathcal{D}$, left and right by the bounds of the interval $I_{0}$ and is of height $\xi^{*}$. 
Adding all rows to the first one it can be written $\chi(M)=\operatorname{det}(\tilde{M}(\lambda))$ with

$$
\tilde{M}(\lambda)=\left(\begin{array}{cccccc}
-\lambda & \cdots & & & \cdots & -\lambda \\
c_{1} & -c_{1}-c_{2}-\lambda & c_{2} & & & \vdots \\
\vdots & c_{2} & \ddots & \ddots & & 0 \\
0 & & \ddots & & & \vdots \\
\vdots & & & & & c_{n-1} \\
c_{n} & \cdots & 0 & \cdots & c_{n-1} & -c_{n-1}-c_{n}-\lambda
\end{array}\right) .
$$

Expanding the determinant along the first row we obtain

$$
\begin{aligned}
\chi(M) & =(-\lambda) \sum_{i=1}^{n}(-1)^{i-1} \operatorname{det}\left([\tilde{M}(\lambda)]_{1 i}\right) \\
& =:(-\lambda) Q(\lambda),
\end{aligned}
$$

where $\operatorname{det}\left([A]_{i j}\right)$ stands for the $(i, j)$-cofactor of $A$. One eigenvalue obviously vanishes and a second eigenvalue, $\lambda_{2}$, is zero if and only if $Q(0)=0$.

We show now that for $i=2, \ldots, n$,

$$
\operatorname{det}\left([\tilde{M}(0)]_{1, i}\right)=-\operatorname{det}\left([\tilde{M}(0)]_{1, i-1}\right)
$$

Let $\mathcal{C}_{k}$ denote the $k^{\text {th }}$ column of matrix $\tilde{M}(0)$ with the first row removed. We write

$$
\begin{aligned}
& \operatorname{det}\left([\tilde{M}(0)]_{1 i}\right)=\left|\begin{array}{lllllll}
\mathcal{C}_{1} & \cdots & \mathcal{C}_{i-2} & \mathcal{C}_{i-1} & \mathcal{C}_{i+1} & \cdots & \mathcal{C}_{n}
\end{array}\right| \\
& =\left|\begin{array}{lllllll}
\mathcal{C}_{1} & \cdots & \mathcal{C}_{i-2} & \sum_{j \neq i} \mathcal{C}_{j} & \mathcal{C}_{i+1} & \cdots & \mathcal{C}_{n}
\end{array}\right|
\end{aligned}
$$

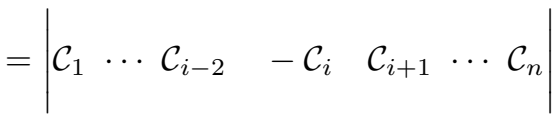

$$
\begin{aligned}
& =-\operatorname{det}\left([\tilde{M}(0)]_{1, i-1}\right) \text {, }
\end{aligned}
$$

where at the second line we used that the determinant is not changed by adding a linear combination of columns to any column, and at the third line, we used the fact that the sum of the elements of any row is zero. We conclude that

$$
\operatorname{det}\left([\tilde{M}(0)]_{1 i}\right)=(-1)^{i-1} \operatorname{det}\left([\tilde{M}(0)]_{11}\right) .
$$


Thus to calculate $Q(0)$ we only have to compute $\operatorname{det}\left([\tilde{M}(0)]_{11}\right)$. Since $[\tilde{M}(0)]_{11}$ is tridiagonal we compute its $L U$-factorization using Thomas algorithm $\underline{40}$,

$$
\begin{aligned}
{[\tilde{M}(0)]_{11} } & =\left(\begin{array}{cccc}
-c_{1}-c_{2} & c_{2} & & \\
c_{2} & -c_{2}-c_{3} & \ddots & \\
& \ddots & \ddots & c_{n-1} \\
& & c_{n-1} & -c_{n-1}-c_{n}
\end{array}\right) \\
& =\left(\begin{array}{cccc}
1 & & 0 & \\
c_{2} / \beta_{1} & 1 & & \\
0 & \ddots & & \ddots \\
c_{n-1} / \beta_{n-2} & 1
\end{array}\right) \cdot\left(\begin{array}{cccc}
\beta_{1} & c_{2} & & \\
& \beta_{2} & \ddots & \\
& & \ddots & c_{n-1} \\
& & & \beta_{n-1}
\end{array}\right),
\end{aligned}
$$

where

$$
\beta_{i}:= \begin{cases}-\left(c_{1}+c_{2}\right), & \text { if } i=1 \\ -\left(c_{i}+c_{i+1}+c_{i}^{2} / \beta_{i-1}\right), & \text { if } i \neq 1\end{cases}
$$

This factorization is only valid for non-singular matrices, but by continuity it can be computed arbitrarily close to points where the determinant vanishes. Computing the determinant of the matrix $[\tilde{M}(0)]_{11}$ then reduces to computing the product of the $\beta_{i}$ 's.

Let us define

$$
\mu_{i}:= \begin{cases}1, & \text { if } i=0 \\ \sum_{j=1}^{i+1} \prod_{\substack{k=1 \\ k \neq j}}^{i+1} c_{k}, & \text { if } i=1, \ldots, n\end{cases}
$$

In Appendix A, we prove by induction that $\mu_{i-1} \cdot \beta_{i}=-\mu_{i}$, for all $i=1, \ldots, n$. This allows to compute the determinant of $[\tilde{M}(0)]_{11}$,

$$
\operatorname{det}\left([\tilde{M}(0)]_{11}\right)=\prod_{i=1}^{n-1} \beta_{i}=(-1)^{n-1} \frac{\mu_{n-1}}{\mu_{0}}=(-1)^{n-1} \sum_{j=1}^{n} \prod_{\substack{k=1 \\ k \neq j}}^{n} c_{k}=(-1)^{n-1} \prod_{j=1}^{n} c_{j} \cdot \sum_{k=1}^{n} c_{k}^{-1},
$$

where the last equality holds as long as all $c_{k}$ 's are nonzero, which is true in the interior of $\mathcal{D}$.

Finally, combining Eqs. (4.10), (4.11) and (4.13) we have

$$
Q(0)=(-1)^{n-1} n \prod_{j=1}^{n} c_{j} \sum_{k=1}^{n} c_{k}^{-1},
$$

and thus

$$
\lambda_{2}=0 \Longleftrightarrow Q(0)=0 \Longleftrightarrow \sum_{k=1}^{n} c_{k}^{-1}=0
$$


Lemma IV.9. For a given value $L \in \mathbb{R}$ and $j \in\left\{j_{0}, j_{1}\right\}$, there is at most one point where $\sum_{k} c_{k}^{-1}=0$ along a connected component of the level curve $\mathcal{A}_{j}=L$.

Remark. From Lemma IV.5 we already know that if $j \notin\left\{j_{0}, j_{1}\right\}, \sum_{k} c_{k}^{-1}$ is never zero.

Proof. We already know that

$$
\sum_{k} c_{k}^{-1}=\frac{\partial \mathcal{A}_{j}}{\partial \varepsilon}
$$

Hence the sum $\sum_{k} c_{k}^{-1}$ equals zero if and only if the level curve of $\mathcal{A}_{j}$ is parallel to the $\varepsilon$ axis. Let us now differentiate the sum $\sum_{k} c_{k}^{-1}$ with respect to $\varepsilon$ at such a point, to see how it varies along the level curve of $\mathcal{A}_{j}$. Using the fact that $c_{j}^{-1}=-\sum_{k \neq j} c_{k}^{-1}$ we have

$$
\begin{aligned}
\frac{\partial}{\partial \varepsilon} \sum_{k=1}^{n} c_{k}^{-1}= & \sum_{k \neq j} \frac{\partial}{\partial \varepsilon}\left[1-\left(\varepsilon+P_{k, k+1}^{*} / K\right)^{2}\right]^{-\frac{1}{2}}-\frac{\partial}{\partial \varepsilon}\left[1-\left(\varepsilon+P_{j, j+1}^{*} / K\right)^{2}\right]^{-\frac{1}{2}} \\
= & \sum_{k \neq j}\left[1-\left(\varepsilon+P_{k, k+1}^{*} / K\right)^{2}\right]^{-\frac{3}{2}}\left[1-\left(\varepsilon+P_{j, j+1}^{*} / K\right)^{2}\right]^{-1} \\
& \times\left[1+\left(\varepsilon+P_{j, j+1}^{*} / K\right)\left(\varepsilon+P_{k, k+1}^{*} / K\right)\right]\left(P_{k, k+1}^{*}-P_{j, j+1}^{*}\right) / K
\end{aligned}
$$

The only term in the last expression that is not necessarily positive is $\left(P_{k, k+1}^{*}-P_{j, j+1}^{*}\right) / K$. But if $j=j_{0}$ (resp. $j=j_{1}$ ), this term is always positive (resp. negative) for $k \neq j$, and consequently the whole sum is positive (resp. negative). Thus, following a connected component of the level curve $\mathcal{A}_{j}=L$, whenever $\sum_{k} c_{k}^{-1}$ hits zero, its derivative always has the same sign, therefore, by continuity, it cannot cross zero more than once. This completes the proof.

The proof of Theorem IV.3 finally relies on Taylor's Lemma $2.1 \frac{11}{}$, which we recall here.

Lemma IV.10 (Taylor 11 ). Let $\left\{\theta_{i}^{(0)}\right\}$ be any stable solution of the power flow Eq. (2.3) on any network. Then for any non-empty node subset $S$,

$$
\sum_{\substack{\langle i j\rangle: \\ i \in S, j \notin S}} \cos \left(\Delta_{i j}^{(0)}\right) \geq 0 .
$$

In other words, if we can partition the nodes of the network in two sets $S$ and $S^{c}$, such that the sum of cosines of the angle differences on all the lines between these two sets is smaller than 0 , then the solution is unstable. In our case of a cycle network, if the angle differences on two lines are larger than $\pi / 2$ or less than $-\pi / 2$, removing these two lines splits the network in two parts, $S$ and $S^{c}$, such that

$$
\sum_{\substack{\langle i j\rangle: \\ i \in S, j \notin S}} \cos \left(\Delta_{i j}^{(0)}\right)<0,
$$

and the solution is unstable. We conclude that there is at most a single $\left|\Delta_{i, i+1}\right|>\pi / 2$. We are now ready to prove Theorem IV.3. 
Remark. Instead of Taylor's Lemma 2.1, we could use the necessary condition for stability of Ref. 41, that if $\left\{\theta_{i}^{(0)}\right\}$ is a stable solution of the power flow Eq. (2.3), then there exists a spanning tree $T$ of the network such that for all edges $e \in E_{T}$,

$$
\cos \left(\Delta_{e}^{(0)}\right) \geq 0 .
$$

Taylor's lemma seems to be slightly more general. As a matter of fact, it is an easy exercise to construct an example of a weighted graph containing a positively weighted spanning tree, but such that there exists a non-empty node subset $S$ with

$$
\sum_{\substack{\langle i j\rangle: \\ i \in S, j \notin S}} \cos \left(\Delta_{i j}^{(0)}\right)<0,
$$

In this case, Taylor's Lemma 2.1 implies instability, while Ref. 41 does not.

Proof of Theorem IV.3. Since for any $K_{0}, \mathcal{A}_{0}\left(K_{0}, \varepsilon\right)$ is an increasing function of $\varepsilon$, we know that for any integer $q \in[-n / 4, n / 4]$, the level set of $\mathcal{A}_{0}=2 \pi q$ is a single level curve. Furthermore, any point on such a level curve corresponds to a stable solution of the power flow Eq. (2.3). Starting from large values of $K$ and following this level curve while decreasing $K$, Corollary IV.6 implies that it meets the boundary of $\mathcal{D}$ at some point. Assume that it meets the upper boundary $\mathcal{D}_{1}$ at $X=\left(K^{*}, \varepsilon^{*}\right)$ as shown on Fig. 4 (the case of the lower boundary $\mathcal{D}_{0}$ is treated in the same way, interchanging $j_{1}$ and $j_{0}$ in what follows). We know that $\mathcal{A}_{0}\left(K^{*}, \varepsilon^{*}\right)=\mathcal{A}_{j_{1}}\left(K^{*}, \varepsilon^{*}\right)$. As $\mathcal{A}_{j_{1}}$ is monotonous on $\mathcal{D}_{1}$ and smooth in the interior of the domain $\mathcal{D}$, there is a level curve of $\mathcal{A}_{j_{1}}=2 \pi q$ starting at $X$ (the red line in Fig. 44), and by Lemma IV.7 it is unique. Furthermore, at this point, the corresponding solution is stable.

According to Corollary IV.6, the level curve of $\mathcal{A}_{j_{1}}$ either meets the boundary of $\mathcal{D}$ or goes to $K \rightarrow \infty$. First, it cannot meet $\mathcal{D}_{1}$ because the value of $\mathcal{A}_{j_{1}}$ is strictly increasing with $K$ on $\mathcal{D}_{1}$ and the level curve cannot be closed by Corollary IV.6. Second, if it goes to $K \rightarrow \infty$, we know from Section IVB that for $K$ large enough, the solution is unstable. Third, if it meets $\mathcal{D}_{0}$, Lemma IV.10 implies that at this point, the corresponding solution is unstable, because at this point, $\left|\Delta_{j_{1}, j_{1}+1}\right|>\pi / 2$ and $\left|\Delta_{j_{0}, j_{0}+1}\right|=\pi / 2$. Thus along the level curve considered, the eigenvalue $\lambda_{2}$ has to change sign. Following Lemmas IV.8 and IV.9 this happens only once, at point $Y$ shown on Fig. 4 where the level curve changes direction with respect to $K$.

Assume now that there is another connected component of the level set of $\mathcal{A}_{j_{1}}=2 \pi q$. From Corollary IV.6 it cannot be closed and by monotonicity of $\mathcal{A}_{j_{1}}$ along $\mathcal{D}_{1}$ and Lemma IV.7, it cannot meet the upper boundary $\mathcal{D}_{1}$. Thus the corresponding solutions are unstable at both ends of this level curve and as, by Lemmas IV.8 and IV.9, $\lambda_{2}$ changes sign at most once along a level curve, then the corresponding solutions are unstable all along this level curve.

We conclude that the number of $\varepsilon$ values corresponding to stable solutions of the power flow equations increases with $K$, because a solution appears at point $Y$ and exists for any larger $K$.

Remark. If there are two indices $i_{1}$ and $i_{2}$ such that $P_{i_{1}, i_{1}+1}^{*}=P_{i_{2}, i_{2}+1}^{*}=P_{\min }^{*}$ (the same works with $\left.P_{\max }^{*}\right)$, then $\sum_{k} c_{k}^{-1}>0$ for $a_{i_{2}} \in(-\pi,-\pi / 2) \cup(\pi / 2, \pi]$ and all other $a_{i}$ 's in $[-\pi / 2, \pi / 2]$, because $c_{i_{1}}=-c_{i_{2}}$ and then

$$
\sum_{k} c_{k}^{-1}=\sum_{k \neq i_{1}, i_{2}} c_{k}^{-1}>0 .
$$

Hence, inside $\mathcal{D}, \lambda_{2}$ never changes sign along the level curves of $\mathcal{A}_{i_{1}}$ and $\mathcal{A}_{i_{2}}$. This result together with the fact that for $K \rightarrow \infty$ the solutions corresponding to the level curves $\mathcal{A}_{i_{1}}$ and $\mathcal{A}_{i_{2}}$ are known to be unstable implies that such solutions remain unstable also for finite values of $K$. Which implies that, in this case, no solution having one angle difference outside the interval $[-\pi / 2, \pi / 2]$ can be locally stable. 
To summarize, we showed that while decreasing $K, \mathcal{N}(K)$ also decreases, and that any stable solution of Eq. (2.3) for finite $K$ is a continuation of a solution for $K \rightarrow \infty$. We also showed that for finite $K$, stable solutions have at most one angle difference outside $[-\pi / 2, \pi / 2]$, and that such solutions are continuations of solutions with all angle differences in $[-\pi / 2, \pi / 2]$. Fig. [5 illustrates the whole situation. The domain $\mathcal{D}$ is bounded above by the curve $\mathcal{D}_{1}$ and below by $\mathcal{D}_{0}$. The blue lines are the $2 \pi q$-level curves of $\mathcal{A}_{0}$ for $q \in\{-1,0,1\}$, i.e. any point on a blue curve gives a pair of values $(K, \varepsilon)$ corresponding to a stable solution of Eq. (2.3) with all angle differenes in $[-\pi / 2, \pi / 2]$. The red dashed lines and the green dash-dotted line are the $2 \pi q$-level curves of $\mathcal{A}_{j_{1}}$ and $\mathcal{A}_{j_{0}}$ respectively. The points on the red dashed curves correspond to solutions (not necessarily stable) where the angle difference on the line carrying $P_{\max }^{*}$ is in $(-\pi,-\pi / 2) \cup(\pi / 2, \pi]$ and the points on the green dash-dotted curve correspond to solutions where the angle difference along the line carrying $P_{\min }^{*}$ is in $(-\pi,-\pi / 2) \cup(\pi / 2, \pi]$. Any blue line meets either a red dashed line on $\mathcal{D}_{1}$ (a zoom-in of this is depicted in Fig. 4) or green dashdotted line on $\mathcal{D}_{0}$. While increasing $K$, stable solutions appear on the level curves of $\mathcal{A}_{j_{1}}$ and $\mathcal{A}_{j_{0}}$ (at point $Y$ in Fig. (4), thus with one angle difference larger than $\pi / 2$ (or less than $-\pi / 2$ ). This angle difference then enters $[-\pi / 2, \pi / 2]$ while $K$ increases. This happens at point $X$ in Fig. [4. Then the stable solution persists for any larger $K$ along the corresponding level curve of $\mathcal{A}_{0}$.

\section{CONCLUSION}

We have investigated the multiplicity of stable stationary solutions to the Kuramoto model. For any network, Theorem III.6 shows that any two different solutions of Eqs. (1.2) and (2.3) differ by a

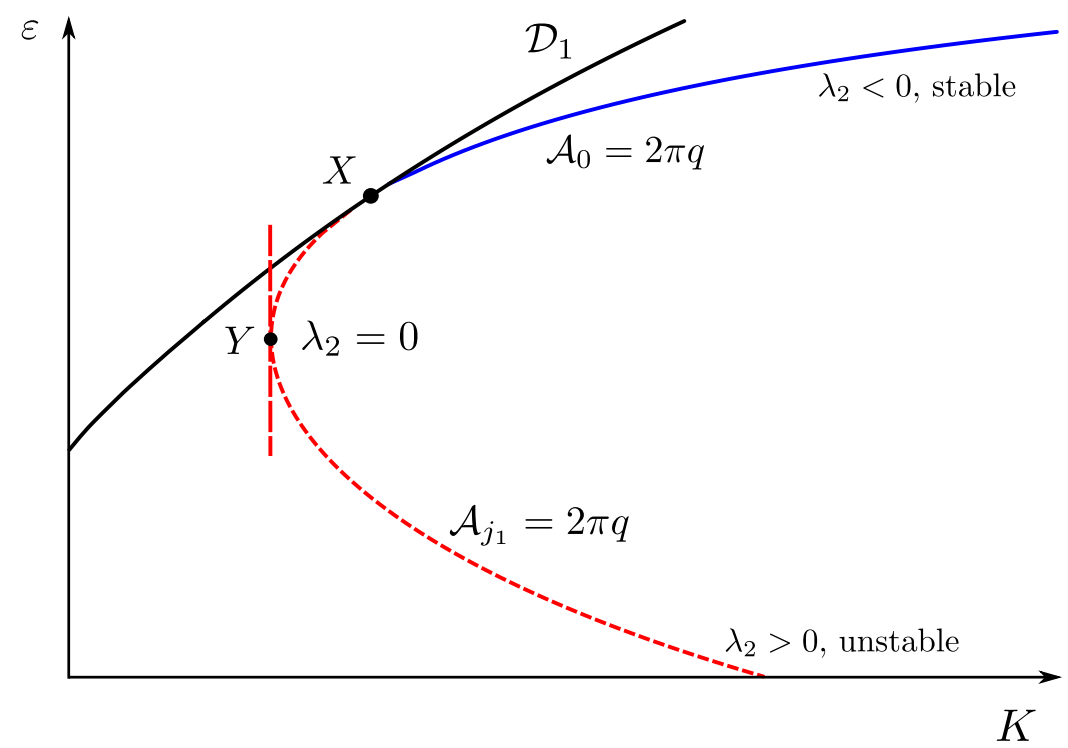

FIG. 4. Level curves $\mathcal{A}_{0}(\varepsilon, K)=2 \pi q$ (blue line) and $\mathcal{A}_{j_{1}}(\varepsilon, K)=2 \pi q$ (dashed red line). Both functions are undefined above the boundary $\mathcal{D}_{1}$ (black line). The corresponding solutions are stable $\left(\lambda_{2}<0\right)$ along the blue curve and between points $X$ and $Y$ on the dashed red curve, and unstable $\left(\lambda_{2}>0\right)$ along the dashed red curve, from $Y$ to $K \rightarrow \infty$. 
combination of circulating flows around the cycles of the network. We showed that these loop flows are quantized and labelled by a topological winding number. In the particular case of single-cycle networks, we then derived an upper bound on the number of stable solutions of the power flow Eq. (2.3),

$$
\mathcal{N} \leq 2 \operatorname{Int}[n / 4]+1
$$

which is algebraic in $n$, the length of the cycle. It significantly improves the exponential bounds obtained if Refs. 17 and 18 . Our result generalizes the bounds obtained by Ochab and Góra ${ }^{28}$, dealing in particular with angle differences larger than $\pi / 2$, and extends the results of Rogge and Aeyles 27 to bidirectional couplings.

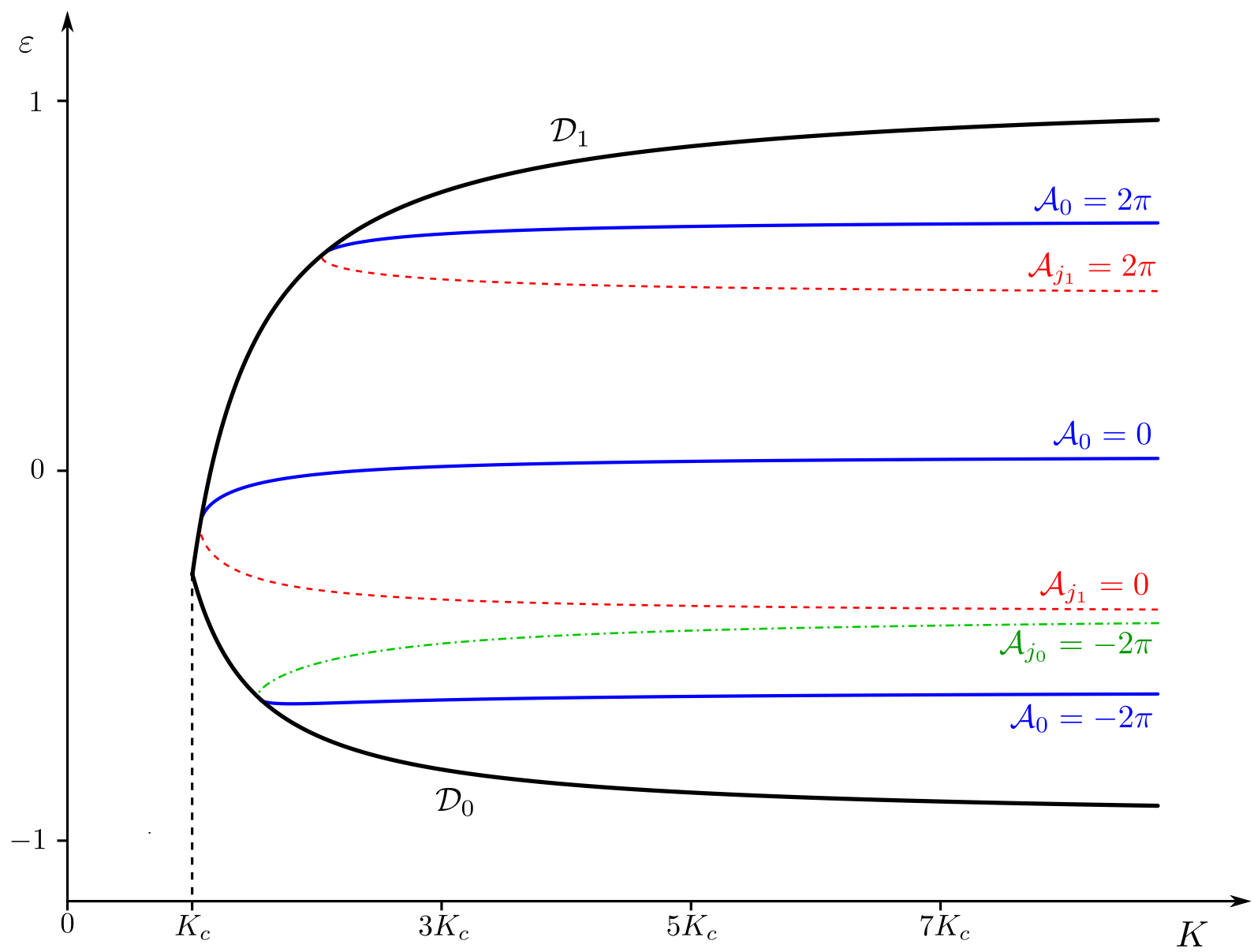

FIG. 5. Level curves $\mathcal{A}_{0}=2 \pi q$ (blue), $\mathcal{A}_{j_{1}}=2 \pi q$ (dashed red) and $\mathcal{A}_{j_{0}}=2 \pi q$ (dash-dotted green), for different $q$-values, in the $(K, \varepsilon)$-plane. The level curves of $\mathcal{A}_{0}$ and $\mathcal{A}_{j_{1}}$ meet on the upper boundary $\mathcal{D}_{1}$ of the domain $\mathcal{D}$ defined in Eq. (4.6), and the level curves of $\mathcal{A}_{0}$ and $\mathcal{A}_{j_{0}}$ meet on the lower boundary $\mathcal{D}_{0}$. The two boundaries $\mathcal{D}_{0}$ and $\mathcal{D}_{1}$ meet at $K_{c}=\left(P_{\max }^{*}-P_{\min }^{*}\right) / 2$. A zoom-in of the region where the level curves $\mathcal{A}_{0}=2 \pi$ and $\mathcal{A}_{j_{1}}=2 \pi$ meet is depicted in Fig. 4 
As parallel results, we obtained some sharp conditions for the solutions on a cycle network with some angle differences in $(-\pi,-\pi / 2) \cup(\pi / 2, \pi]$ to be stable. We showed that at most one angle difference can be larger than $\pi / 2$ in a stable solution and it can only be the case on the most loaded line. Moreover, any stable solution with an angle difference larger than $\pi / 2$ can be directly connected to a solution with all angle differences in $[-\pi / 2, \pi / 2]$ for the same network at larger $K$.

The quantized loop flows discussed above are highly undesirable in electric power grids. They transmit power which is never distributed but only generates ohmic losses. A deeper understanding of loop flows, how they appear and how to make them disappear could greatly help in devising power grids protected against their emergence. In all likelihood, this would be of great interest for power grid operators.

Another line of possible future research would be to compare the stability of different solutions. This could be done in at least two ways, first, comparing the spectra of the stability matrices for different solutions, second, comparing the volumes of the respective basins of attraction. This second approach was proposed in Ref. 31. Of particular interest would be to relate these two measures of stability with winding numbers.

Obviously, the next step in this investigation is to study how our results can be extended to more general networks with multiple cycles. It is clear that in the case of independent cycles as in Fig. 6a, the number of stable solutions is bounded by

$$
\mathcal{N}=\left(2 \operatorname{Int}\left[n_{1} / 4\right]+1\right)\left(2 \operatorname{Int}\left[n_{2} / 4\right]+1\right),
$$

where $n_{1}$ and $n_{2}$ are the number of edges in the two cycles respectively. In this case, a loop flow on one of the cycles does not influence the loop flow on the other cycle. The problem becomes more intricate when we have to deal with cycles sharing edges, where the loop flows add, Fig. 6b. Here the flow on one of the cycles limits the loop flow on the other cycle because it could saturate the capacity of the common lines. Work along those lines is in progress.

\section{ACKNOWLEDGMENTS}

This work has been supported by the Swiss National Science Foundation under an AP Energy Grant. The authors would like thank L. Pagnier for useful discussions.

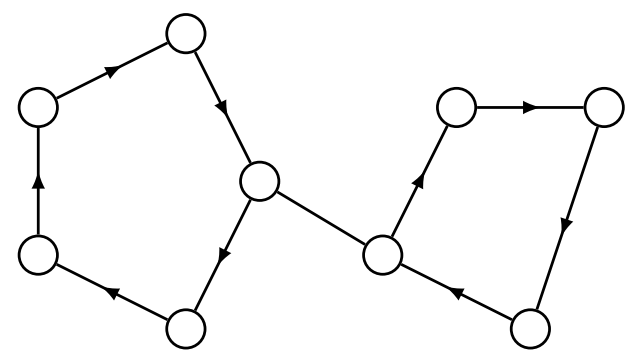

(a)

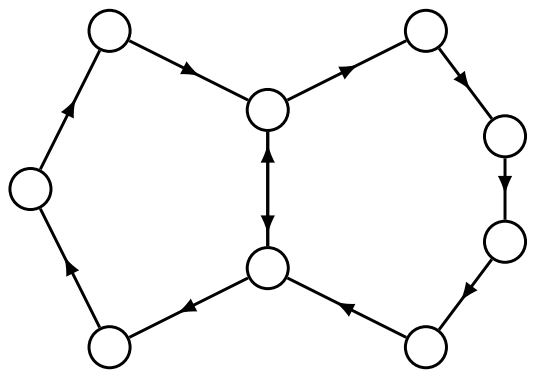

(b)

FIG. 6. Two graphs with two cycles. Left: the cycles are independent but connected, the loop flow on one of them does not influence the loop flow on the other one. Right: the cycles are not independent. Thus having a loop flow on one of them restricts the possible flows on the other one. 


\section{Appendix A $L U$-DECOMPOSITION OF THE STABILITY MATRIX}

We prove inductively that the diagonal elements $\beta_{i}$ of the upper triangular matrix of the $L U$ factorization of the stability matrix, obtained through Thomas algorithm 40 , satisfy the relation

$$
\mu_{i-1} \cdot \beta_{i}=-\mu_{i}
$$

where $\mu_{i}$ 's are defined in Eq. (4.12). For $i=1$, we have

$$
\begin{aligned}
\mu_{0} \cdot \beta_{1} & =1 \cdot\left(-c_{1}-c_{2}\right) \\
& =-\mu_{1} .
\end{aligned}
$$

Suppose now that $\mu_{i-1} \cdot \beta_{i}=-\mu_{i}$. Let us show that $\mu_{i} \cdot \beta_{i+1}=-\mu_{i+1}$.

$$
\begin{aligned}
\mu_{i} \cdot \beta_{i+1} & =\mu_{i}\left(-c_{i+1}-c_{i+2}-\frac{c_{i+1}^{2}}{\beta_{i}}\right) \\
& =-c_{i+1} \mu_{i}-c_{i+2} \mu_{i}+c_{i+1}^{2} \mu_{i-1} \\
& =-c_{i+1} \sum_{j=1}^{i+1} \prod_{\substack{k=1 \\
k \neq j}}^{i+1} c_{k}-c_{i+2} \sum_{j=1}^{i+1} \prod_{\substack{k=1 \\
k \neq j}}^{i+1} c_{k}+c_{i+1}^{2} \sum_{j=1}^{i} \prod_{\substack{k=1 \\
k \neq j}}^{i} c_{k} \\
& =-c_{i+1}\left(c_{i+1} \sum_{j=1}^{i} \prod_{\substack{k=1 \\
k \neq j}}^{i} c_{k}+\prod_{k=1}^{i} c_{k}\right)-c_{i+2} \sum_{j=1}^{i+1} \prod_{\substack{k=1 \\
k \neq j}}^{i+1} c_{k}+c_{i+1}^{2} \sum_{j=1}^{i} \prod_{\substack{k=1 \\
k \neq j}}^{i} c_{k} \\
& =-c_{i+1} \prod_{k=1}^{i+1} c_{k}-c_{i+2} \sum_{j=1}^{i+1} \prod_{\substack{k=1 \\
k \neq j}}^{i+1} c_{k} \\
& =-\sum_{j=1}^{i+2} \prod_{\substack{k=1 \\
k \neq j}}^{i+2} c_{k} \\
& =-\mu_{i+1} .
\end{aligned}
$$

${ }^{1}$ S. H. Strogatz, SYNC, the Emerging Science of Spontaneous Order (Penguin Books, London, 2003).

${ }^{2}$ A. T. Winfree, J. Theoret. Biol. 16, 15 (1967).

${ }^{3}$ Y. Kuramoto, Progr. Theoret. Phys. Suppl. 79, 223 (1984).

${ }^{4}$ S. H. Strogatz, Physica D 143, 1 (2000).

${ }^{5}$ J. A. Acebrón, L. L. Bonilla, C. J. Pérez Vicente, F. Ritort, and R. Spigler, Rev. Mod. Phys. 77, 137 (2005).

${ }^{6}$ F. Dörfler and F. Bullo, Automatica 50, 1539 (2014).

${ }^{7}$ G. B. Ermentrout, J. Math. Biol. 22, 1 (1985).

${ }^{8}$ J. L. van Hemmen and W. F. Wreskinski, J. Stat. Phys. 72, 145 (1993).

${ }^{9}$ D. Aeyels and J. A. Rogge, Prog. Th. Phys. 112, 921 (2004).

${ }^{10}$ R. E. Mirollo and S. H. Strogatz, Physica D 205, 249 (2005).

${ }^{11}$ R. Taylor, J. Phys. A 45, 055102 (2012)

${ }^{12}$ A. J. Korsak, IEEE Trans. Power App. Syst. PAS-91, 1093 (1972)

${ }^{13}$ A. R. Bergen and V. Vittal, Power Systems Analysis (Prentice Hall, 2000).

${ }^{14}$ Y. Tamura, H. Mori, and S. Iwamoto, IEEE Trans. Power App. Syst. PAS-102, 1115 (1983)

${ }^{15}$ A. Klos and J. Wojcicka, Int. J. Elect. Power Energy Syst. 13, 268 (1991)

${ }^{16}$ J. Baillieul and C. I. Byrnes, IEEE Trans. Circuits Syst. 29, 724 (1982). 
${ }^{17}$ H. D. Nguyen and K. S. Turitsyn, in PES General Meeting - Conference Exposition, 2014 IEEE (2014).

${ }^{18}$ D. Mehta, N. Daleo, F. Dörfler, and J. D. Hauenstein, Chaos 25 (2015)

${ }^{19}$ N. Janssens and A. Kamagate, Int. J. Elect. Power Energy Syst. 25, 591 (2003)

${ }^{20}$ L. Onsager, Nuovo Cimento 6, 249 (1949).

${ }^{21}$ R. P. Feynman, Progress in Low Temperature Physics 1, 34 (1955).

${ }^{22}$ A. A. Abrikosov, Sov. Phys. JETP 5, 1174 (1957).

${ }^{23}$ N. Byers and C. N. Yang, Phys. Rev. Lett. 7, 46 (1961).

${ }^{24}$ K. A. Matveev, A. I. Larkin, and L. I. Glazman, Phys. Rev. Lett. 89, 096802 (2002).

${ }^{25}$ G. Rastelli, I. M. Pop, and F. W. J. Hekking, Phys. Rev. B 87, 174513 (2013).

${ }^{26}$ W. A. Bukhsh, A. Grothey, K. I. M. McKinnon, and P. A. Trodden, IEEE Trans. Power Syst. 28, 4780 (2013)

${ }^{27}$ J. A. Rogge and D. Aeyels, J. Phys. A 37, 11135 (2004)

28 J. Ochab and P. F. Góra, Acta Phys. Pol. B Proc. Suppl. 3, 453 (2010).

${ }^{29}$ P. F. C. Tilles, F. F. Ferreira, and H. A. Cerdeira, Phys. Rev. E 83 (2011)

${ }^{30}$ T. K. Roy and A. Lahiri, Chaos, Solitons \& Fractals 45, 888 (2012)

${ }^{31}$ D. A. Wiley, S. H. Strogatz, and M. Girvan, Chaos 16, 015103 (2006)

${ }^{32}$ P. W. Anderson, Rev. Mod. Phys. 38, 298 (1966)

${ }^{33}$ G. Blatter, M. V. Feigel'man, V. B. Geshkenbein, A. I. Larkin, and V. M. Vinokur, Rev. Mod. Phys. 66, 1125 (1994)

${ }^{34}$ R. Fazio and H. van der Zant, Physics Reports 355, 235 (2001)

${ }^{35}$ C. J. Tavora and O. J. M. Smith, IEEE Trans. Power App. Syst. PAS-91, 1138 (1972)

${ }^{36}$ R. A. Horn and C. R. Johnson, Matrix Analysis (Cambridge University Press, New York, 1986).

${ }^{37}$ F. Dörfler, M. Chertkov, and F. Bullo, Proceedings of the National Academy of Sciences 110, 2005 (2013)

${ }^{38}$ N. Biggs, Algebraic graph theory, 2nd ed. (Cambridge University Press, 1993).

${ }^{39}$ W. Cheney, Analysis for Applied Mathematics (Springer, New York, 2001).

${ }^{40}$ W. Gander, M. J. Gander, and F. Kwok, Scientific Computing - An Introduction using Maple and MATLAB (Springer International Publishing, 2014).

${ }^{41}$ A.-L. Do, S. Boccaletti, and T. Gross, Phys. Rev. Lett. 108 (2012). 